\title{
ETUDE LIMNOLOGIQUE \\ DU BASSIN VERSANT DU LAC SAINT-JEAN (QUEBEC, CANADA). CORRESPONDANCE ENTRE PRODUCTION ET BIODISPONIBILITE D'ELEMENTS NUTRITIFS
}

\author{
par P. COUTURE 1 , D. CLUIS ${ }^{1}$ et L. POTVIN ${ }^{1}$
}

La connaissance de la distribution des superficies occupées par la forêt et des surfaces exploitées à des fins agricoles sur le bassin versant du lac Saint-Jean permet de distinguer deux grands groupes de sous-bassins, les tributaires forestiers et les rivières agricoles. Les charges calculées en substances nutritives (azote, phosphore), les niveaux d'enrichissements mesurés en substances de croissances potentiellement disponibles pour le phytoplancton (potentiel de fertilité (PF) ) et les concentrations en chlorophylle- $a$ dosées sont la résultante de l'utilisation et de l'état des territoires drainés. Une typologie des tributaires étudiés est proposée en confrontant les charges calculées $(\mathrm{N} / \mathrm{P})^{2}$ et la mesure de leur biodisponibilité potentielle pour le phytoplancton $(\mathrm{PF})$ :

$$
\begin{aligned}
N / P_{(\text {forestior) }} & >N / P_{\text {(agricole) }} \\
P F_{(\text {forestier) }} & <P F_{(\text {agricole) }}
\end{aligned}
$$

\section{Limnological study of lake St. John watershed (Québec, Canada). Relationship between production and bioavailability of nutrients.}

The lake St. John watershed has been subdivided into two land-use types on the basis of the areal distribution of forests and agricultural lands. The tributaries of each watershed were characterized in terms of nitrogen and phosphorous loadings, potential primary productivity, and concentrations of chlorophyll'a'. From the calculated loading ratios (N/P) and their potential "bioavailability" (PF) to phytoplankton, a typology of the major tributaires has been proposed :

$$
\begin{aligned}
N / P_{\text {(rorest) }} & >N / P_{\text {(agricul tural) }} \\
P F_{\text {(rorest) }} & <P F_{\text {(agri eul tural) }}
\end{aligned}
$$

\section{1. - INTRODUCTION}

Les caractéristiques géographiques d'un bassin hydrographique exercent une influence sur la qualité du milieu aquatique. Parmi ces caractéristiques, il y a celles qui concernent le milieu naturel : topographie, géologie, végétation, etc., et celles qui sont liées aux activités

1. Institut national de la recherche scientifique (INRS-Eau), C.P. 7500, SainteFoy, Québec, Canada G1V 4C7.

2. Le rapport $\mathrm{N} / \mathrm{P}$ est obtenu à partir d'éléments calculés et non mesurés. 
humaines : urbanisation, agriculture, industrie, etc. Ce sont toutefois ces dernières qui engendrent les véritables problèmes de détérioration de la qualité des eaux en contribuant à l'accélération du processus d'eutrophisation des écosystèmes aquatiques (Lee 1973; Lee et al. 1978; Likens et Loucks 1978).

La relation [caractéristiques géographiques $\rightarrow$ qualité du milieu aquatique] a été exploitée abondamment dans la conception de modèles reliant l'utilisation du territoire à la teneur en éléments nutritifs des eaux d'un bassin versant.

Ces modèles permettent, par exemple, de calculer les charges en azote et en phosphore transitées à l'intérieur de l'écosystème aquatique à partir d'une connaissance de l'état ou encore de l'utilisation des territoires drainés (Cluis et al. 1979; Dillon et Kirchner 1975; Dillon et Rigler 1975 ; Kirchner 1975 ; Patalas et Salki 1973; Vollenweider 1968).

Le but de cette étude est de démontrer l'influence de certaines caractéristiques géographiques (forêt, agriculture) du bassin versant du lac Saint-Jean sur l'enrichissement des tributaires en substances biodisponibles pour les producteurs primaires. Les niveaux d'enrichissements sont déterminés à l'aide de la mesure du potentiel de fertilité (bioessai : Selenastrum capricornutum). Ce test biologique, largement utilisé dans plusieurs pays, a été employé à l'intérieur de travaux portant aussi bien sur le contrôle de la qualité de l'environnement aquatique que sur la gastion de cette ressource. Les études concernant les substances nutritives, effectuées à l'aide du potentiel de fertilité ( $\mathrm{PF}$ ), peuvent être séparées en trois catégories.

Les premières ont consisté à appliquer le test à des produits d'usage domestique. Les résultats obtenus ont été discutés en fonction de considérations environnementales comme, par exemple, les effets de l'utilisation des détergents sur le milieu naturel (Mitchell et Buzzell 1971 ; Payne 1973 ; Sturm et Payne 1973).

Les secondes ont utilisé la technique du PF pour caractériser des niveaux trophiques ou encore pour identifier les nutriments contrôlant la production primaire (Berland et al. 1974, 1976; Doemel et Brooks 1975; Francisco et Weiss 1973; Payne 1975; Robarts et Southall 1977; Steyn et al. 1974 ; Thomas et al. 1974; Weiss 1975). Dans cette catégorie, quelques travaux ont établi une relation significative entre le niveau trophique de différents lacs et la mesure du PF (Chiaudani et Vighi 1974; Miller et al. 1974) alors que d'autres, après avoir établi l'état nutritionnel d'un réservoir, en estimaient le niveau trophique (Toerien et Steyn 1973). Des mesures du PF effectuées sur des échantillons prélevés en milieu lotique (Greene et al. 1975 a) ont aussi été caractérisées à partir des indices de productivité développés pour les lacs par Miller et al. (1974). 
La troisième catégorie de travaux a concerné une préoccupation importante des différents utilisateurs de ce type de bioessais à savoir comment des tests biologiques effectués en laboratoire sur des eaux naturelles pouvaient refléter les conditions trouvées dans l'environnement (Greene et al. $1975 \mathrm{~b}$ ). Les chercheurs ont tenté de répondre à cette question en associant les mesures du PF à deux paramètres capables de mesurer la production primaire du milieu : la biomasse phytoplanctonique et les concentrations en chlorophylle- $a$. Les résultats obtenus ont suggéré une relation significative $(r>0,93)$ entre les mesures du PF effectuées au laboratoire et celles faites dans l'environnement naturel (Greene et al. 1975 b, 1976 a, 1976 b; Mahoney 1973).

Tous ces travaux ont suggéré que la mesure du PF est non seulement représentative des constituants abiotiques du système, mais aussi de ses constituants biotiques. Nous sommes cependant d'avis que ce paramètre ne peut déterminer les relations écologiques entre les populations d'algues du milieu naturel (Gerhold et Otto 1976), mais il n'en reste pas moins qu'il se révèle un excellent outil d'appréciation des niveaux de production de l'environnement aquatique. A cet effet, ce type de bioessai représente une approche raisonnable pour quantifier les niveaux d'enrichissement d'un système aquatique (Middlebrooks et al. 1971).

\section{2. - MATERIEL ET METHODE}

\section{1. - Description du territoire}

Le bassin versant du lac Saint-Jean a une superficie d'environ $73000 \mathrm{~km}^{2}$; il est en totalité situé à l'intérieur de la région hydrographique 06 du ministère des Richesses naturelles du Québec (fig. 1). La majeure partie du territoire s'étend sur les hautes-terres du Bouclier canadien et est recouverte de forêt de type boréal. La partie défrichée du bassin, là où les activités humaines sont concentrées, ne représente que $8 \%$ de la superficie totale et correspond essentiellement à des basses-terres formant une enclave au sein du Bouclier canadien.

Le lac Saint-Jean, qui occupe une superficie d'environ $1000 \mathrm{~km}^{2}$, sert de collecteur à de nombreuses rivières prenant leur source dans le Bouclier canadien. Douze de ces tributaires, les plus représentatifs à notre connaissance de la qualité des eaux du bassin versant, ont été sélectionnés pour cette étude.

La plus considérable de ces rivières est la Péribonca, qui se jette au nord-est du lac; d'une longueur de $480 \mathrm{~km}$, elle draine un bassin de $26900 \mathrm{~km}^{2}$. Au nord-ouest du lac, la Mistassini, d'une longueur de 


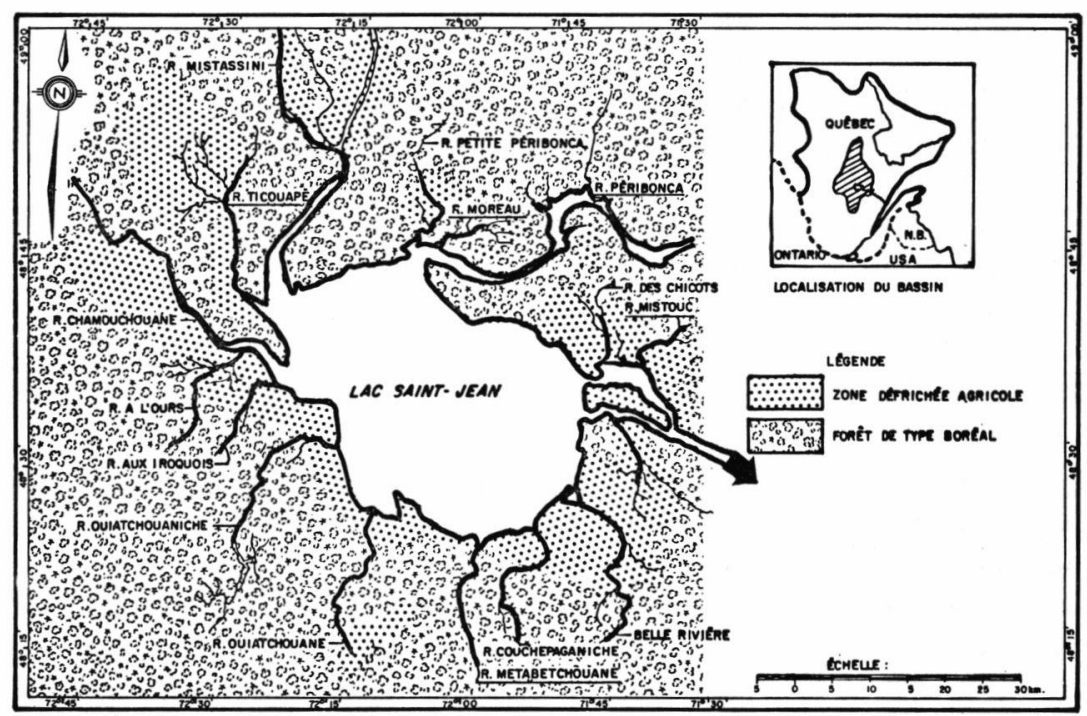

Fig. 1. - Le lac Saint-Jean et son bassin versant: principaux tributaires et utilisation du sol.

$320 \mathrm{~km}$, draine un territoire de $21900 \mathrm{~km}^{2}$. La Chamouchouane, d'une longueur de $320 \mathrm{~km}$, draine une superficie de $15700 \mathrm{~km}^{2}$ et se déverse au nord-ouest du lac, à proximité de l'embouchure de la Mistassini. A eux seuls, ces trois grands tributaires du nord drainent un territoire représentant $87 \%$ de la superficie totale du bassin. Les rivières Ticouapé, Petite Péribonca, Moreau, des Chicots et Mistouc viennent compléter l'étude des tributaires de la section nord du bassin versant. Quant aux tributaires du sud, ils sont de moindre importance. La rivière Métabetchouane est la rivière qui draine la plus grande superficie au sud du lac, soit $2300 \mathrm{~km}^{2}$. Les autres rivières considérées dans cette région sont la Couchepaganiche, la Ouiatchouane et la Ouiatchouaniche.

\section{2. - Echantillonnage}

Dans le cadre d'une étude plus globale (INRS-Eau 1980) l'échantillonnage fut effectué à partir des ponts routiers situés sur les tributaires, à proximité de leurs confluences avec le lac. Quatre campagnes de prélèvement furent effectuées : 22 mai, 17 juin, 15 juillet et 23 septembre 1975.

La technique de prélèvement des échantillons destinés à la détermination du PF consistait à obtenir un échantillon représentatif de la colonne d'eau à l'endroit du prélèvement; à cette fin, l'opération consistait à remplir les bouteilles à l'aide d'un hydro-capteur, en effec- 
tuant un mouvement alternatif de la surface vers le fond et vice versa. Pour les échantillons destinés à la détermination des concentrations en chlorophylle, les prélèvements ont été faits en surface.

Les échantillons d'eau étaient par la suite acheminés vers les laboratoires de l'INRS-Eau, à Québec. Les bouteilles destinées aux expériences de fertilité étaient remplies à capacité maximale et acheminées $\left(4^{\circ} \mathrm{C}\right.$, obscurité) au laboratoire; les échantillons réservés au dosage de la chlorophylle étaient d'abord filtrés et seules les membranes étaient transportées $\left(4^{\circ} \mathrm{C}\right.$, obscurité) vers Québec.

\section{3. - Mesure du PF et dosage de la chlorophylle}

La mesure du potentiel de fertilité a été effectuée selon la méthode développée par l'Environmental Protection Agency des Etats-Unis (EPA 1971). Un échantillon composite (eau non filtrée) est stérilisé et une souche d'algues (Selenastrum capricornutum) y est ensemencée à raison de 5000 algues $/ \mathrm{ml}$. La souche d'algues utilisée provient du Pacific Northwest Water Laboratory (Corvallis, Oregon). La souche est repiquée toutes les semaines et seules les cultures en phase de croissance exponentielle servent d'inoculum. Les conditions d'incubation des échantillons d'eau sont les suivantes: 16 heures de photopériode, $5400 \mathrm{lux}, 24 \pm 2{ }^{\circ} \mathrm{C}$. Les échantillons inoculés sont agités à la main tous les jours et la population d'algues est mesurée à la fin de la période d'incubation ( 21 jours) à l'aide d'un compteur de particules (Coulter Counter Model ZB, cellule de $100 \mu$ ) ; la valeur ainsi obtenue s'exprime en quantité de particules par millilitre. Cette valeur (nombre de cellules $/ \mathrm{ml}$ ) est par la suite transformée en biomasse à l'aide d'une constante (INRS-Eau 1976). Les mesures ont été effectuées en triplicata et les résultats non reproductibles à moins de $20 \%$ ont été éliminés.

Le dosage de la chlorophylle a été effectué selon la technique décrite par Parsons et Strickland (1972) ; cette méthode permet de doser la chlorophylle- $a$ totale présente dans l'échantillon d'eau.

\section{4. - Calcul des apports}

Les charges en éléments nutritifs ont été calculés à l'aide d'un modèle d'apport développé conjointement par le ministère des Richesses naturelles du Québec et l'INRS-Eau (Cluis et al. 1979; MRN 1977). La technique utilisée permet de lier l'état d'un territoire ou encore ses statistiques d'utilisation aux quantités d'azote et de phosphore transitées. Les données nécessaires aux calculs des charges apparaissent au tableau 1; elles proviennent d'estimations cartographiques et de 
Tableau I. - Utilisation du sol dans les sous-bassins du lac Saint-Jean.

\begin{tabular}{|c|c|c|c|c|c|c|c|c|c|c|c|c|c|c|}
\hline & \multirow{2}{*}{$\begin{array}{c}\text { SUPERFICIE } \\
\text { TOTMLE } \\
\text { ow EASSIK } \\
\mathrm{km}^{2}\end{array}$} & \multicolumn{2}{|c|}{ Foret } & \multicolumn{2}{|c|}{ AGR ICUL TURE } & \multicolumn{3}{|c|}{ POPLATIIOM } & \multirow{2}{*}{$\begin{array}{l}\text { Bovins } \\
\text { tetes }\end{array}$} & \multirow{2}{*}{$\begin{array}{l}\text { macs } \\
\text { cetes }\end{array}$} & \multirow{2}{*}{$\begin{array}{l}\text { ELEVAGE } \\
\text { MouTous } \\
\text { tetes }\end{array}$} & \multirow{2}{*}{$\begin{array}{c}\text { pouks ET } \\
\text { poinets } \\
\text { tetes }\end{array}$} & \multirow{2}{*}{$\begin{array}{c}\text { SUPERFICIE } \\
\text { FERTIEISEE } \\
\text { PAR EMERAIS } \\
\text { CHIMI Gives } \\
\text { Koj }\end{array}$} & \multirow{2}{*}{$\begin{array}{l}\text { IMOUSTRIE } \\
\text { cilimewialat } \\
\text { nodbre } \\
\text { d'eployes }\end{array}$} \\
\hline & & $k^{2}$ & 3 & $t^{2}$ & 8 & nob. & hab. & now. & & & & & & \\
\hline \multicolumn{15}{|c|}{ 4) rivieres forestiêres } \\
\hline Meribonce & 26925 & 26698 & 99.1 & 16 & 0.1 & 94 & 1678 & 2622 & 1179 & 154 & 356 & 1266 & 1.06 & - \\
\hline Miscessint & 21871 & 21650 & 90.9 & 6 & 0.4 & 12655 & 1712 & 14427 & 486 & 762 & 415 & 13825 & 8.21 & - \\
\hline Chenowernousere & is431 & 15275 & 98.9 & s1 & 0.4 & 6127 & 1958 & 0005 & 3961 & 599 & 53 & 21565 & 3.11 & 43 \\
\hline Petite Peribonce & 1165 & 110 & 96.9 & 32 & 2.0 & 250 & 1140 & 1900 & 2372 & 102 & 86 & 4281 & 2.03 & - \\
\hline netabe tehouine & 2325 & 2301 & 98.9 & 14 & 0.6 & 969 & 1009 & 1978 & 1064 & 20 & 20 & 497 & 0.41 & $70^{\circ}$ \\
\hline Ouldtchowenne & 955 & 926 & 96.9 & 7 & 0.7 & 1955 & 103 & 2050 & 280 & 22 & 36 & 569 & 0.16 & - \\
\hline Qulatchowaniche & 352 & 323 & 91.7 & 12 & 3.0 & 0 & 2492 & 2492 & 901 & 120 & 99 & 14287 & 0.36 & - \\
\hline \multicolumn{15}{|l|}{ b) riviteres agricoles } \\
\hline Ileoupe & 665 & 259 & 36.9 & 274 & 41 & 3199 & 6612 & 9en1 & 20764 & 4206 & $14 n$ & 70044 & 29.73 & $\infty$ \\
\hline Nistowe & 215 & 107 & 49.7 & 53 & 24 & 700 & 2075 & 2775 & 1195 & 1090 & 934 & 2014 & 2.75 & - \\
\hline Des onlcots & 51 & 33 & 64.7 & 16 & 31 & 0 & 659 & 659 & $13 n$ & 483 & 378 & 2262 & 1.30 & - \\
\hline Moreau & I11 & $n$ & 64.8 & 19 & 17 & 0 & 651 & 651 & 1821 & 42 & 330 & 1154 & 2.67 & $=$ \\
\hline Couchepaguniche & 100 & 70 & 70 & 22 & 22 & 1347 & 154 & 1501 & 2017 & 306 & 74 & 23812 & 1.03 & c \\
\hline
\end{tabular}

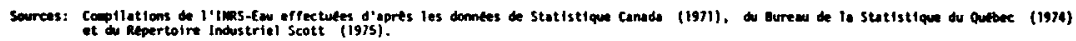

statistiques municipales extraites du recensement de 1971 qui ont été regroupées et ventilées proportionnellement à la superficie des sousbassins. Pour chacun des apports (égouts domestiques, rejets industriels, ruissellement, élevage, précipitations, forêts), les productivités spécifiques en azote et en phosphore de chaque type de producteurs ont été établies à partir d'une étude bibliographique (MRN 1977), en séparant les apports ponctuels à la rivière des apports diffus, c'est-àdire non totalement transférés (Tableau II).

Tableau II. - Apports spécifiques moyens annuels.

\begin{tabular}{|c|c|c|c|c|c|c|}
\hline & PRooucteun & TTPES O'NPPORTS & & NPPORTS EW N & & NPORTS EW \\
\hline Aports neturels & $\begin{array}{l}\text { Lacs - plute sur le lac } \\
\text { forets - rulssellemt } \\
\text { morecages - rulssellement } \\
\text { sol m - plule sur sol nu }\end{array}$ & $\begin{array}{l}\text { reels } \\
\text { potentiels } \\
\text { reels } \\
\text { potentiels }\end{array}$ & & $\begin{array}{l}k g / \text { Jowr }-\left.k^{2}\right|^{2} \\
k g / \text { Jour }-k^{2} m^{2} \\
k / \text { Jour } k m^{2} \\
k g / \text { Jour-kon }\end{array}$ & $\begin{array}{l}0.02 \\
0.6 \\
0.08\end{array}$ & 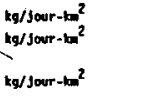 \\
\hline $\begin{array}{l}\text { Apports de la } \\
\text { population hum ine }\end{array}$ & $\begin{array}{l}\text { population svec gout } \\
\text { population sans ggout }\end{array}$ & $\begin{array}{l}\text { reels } \\
\text { potentiels }\end{array}$ & $\begin{array}{l}14 \\
14\end{array}$ & $\begin{array}{l}\text { g/jour-habit tant } \\
\text { g/jour-habl tant }\end{array}$ & $\begin{array}{l}1.0 \\
1.0\end{array}$ & $\begin{array}{l}\text { g/Jour-habltant } \\
\text { g/Jour-habitant }\end{array}$ \\
\hline $\begin{array}{l}\text { Apports de is } \\
\text { population antinele }\end{array}$ & $\begin{array}{l}\text { bowins } \\
\text { porcs } \\
\text { movtons } \\
\text { poulets } \\
\text { chevoux }\end{array}$ & $\begin{array}{l}\text { potentiels } \\
\text { potentiels } \\
\text { potentiels } \\
\text { potentiels } \\
\text { potentiels }\end{array}$ & $\begin{array}{r}107 \\
31 \\
24 \\
2 \\
159\end{array}$ & $\begin{array}{l}9 / \text { Jour-enimal } \\
9 / \text { Jour-entalal } \\
\text { g/Jour-entimal } \\
\text { g/jour-animal } \\
\text { g/Jour-antiand }\end{array}$ & $\begin{array}{r}33.5 \\
9.5 \\
4.4 \\
0.7 \\
23.1\end{array}$ & 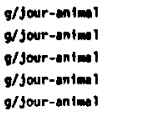 \\
\hline $\begin{array}{l}\text { Apports des } \\
\text { engrels chiniques }\end{array}$ & $\begin{array}{l}\text { ble } \\
\text { ovoine } \\
\text { orge } \\
\text { frults de vergers } \\
\text { petits fruits } \\
\text { Daturage defriche } \\
\text { foin cultive } \\
\text { mils-grath } \\
\text { tabac } \\
\text { patutes } \\
\text { betteraves } \\
\text { legumes } \\
\text { sutres }\end{array}$ & $\begin{array}{l}\text { potentiels } \\
\text { potentiels } \\
\text { potentiels } \\
\text { potentiels } \\
\text { potentiels } \\
\text { potentiels } \\
\text { potentiels } \\
\text { potentiels } \\
\text { potentiels } \\
\text { potentiels } \\
\text { potentiels } \\
\text { potentiels } \\
\text { potentiels }\end{array}$ & $\begin{array}{l}15 \\
4.6 \\
12 \\
3.4 \\
29 \\
15.4 \\
15.4 \\
40 \\
11 \\
25 \\
25 \\
15 \\
31\end{array}$ & 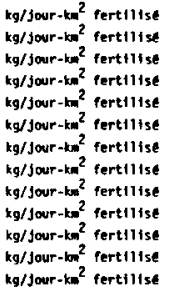 & $\begin{array}{c}9.4 \\
9.4 \\
12 \\
7.1 \\
28 \\
9.1 \\
9.1 \\
13 \\
18 \\
23 \\
24 \\
12 \\
11\end{array}$ & 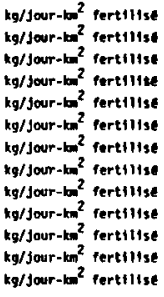 \\
\hline
\end{tabular}


La charge $F$ à l'embouchure de chaque rivière est calculée à partir des productions ponctuelles ou diffuses et d'un schéma de drainage qui a été préalablement établi à l'aide d'une méthode de carreaux utilisant comme support cartographique le quadrillage Universel Transverse de Mercator. Deux coefficients de transfert sont définis :

$\mathrm{C}_{\mathrm{I}}=$ coefficient interne de transfert des apports potentiels au cours d'eau ;

$\mathrm{C}_{\mathrm{r}}=$ coefficient de transport de carreau en carreau dans la rivière.

Si on note $D_{1}$ et $P_{i}$ les apports diffus et ponctuels d'un élément « $i$ » pour un carreau situé à $\mathrm{k}$ carreaux en amont d'un point de contrôle, la contribution de ce carreau à la charge transitée pour une période de temps donnée, à la station, s'écrit :

$$
\mathrm{F}_{\mathrm{i}}=\mathrm{C}_{\mathrm{r}}{ }^{\mathrm{k}} \times\left(\mathrm{P}_{\mathrm{i}}+\mathrm{C}_{\mathrm{I}} \cdot \mathrm{D}_{\mathrm{i}}\right)
$$

Le transport total en éléments nutritifs à la station est donc la somme des contributions de ce type pour l'ensemble des carreaux situés à l'amont. Ainsi, ce schéma permet de simuler en tout point du réseau hydrographique les charges transportées. La signification physique des coefficients du modèle est la suivante :

- $C_{I}$ représente la fraction des apports potentiels atteignant la rivière;

- $\mathrm{C}_{\mathrm{T}}$ représente le transport des charges une fois acheminées dans le cours d'eau.

Dans le cas du bassin du lac Saint-Jean, une version simplifiée de ce modèle a été utilisée. Il s'agit de l'utilisation du modèle sur une base annuelle, sans échantillonnage de contrôle pour évaluer les apports à l'exutoire de chaque bassin versant.

Sur une base annuelle et pour une année hydrologique moyenne, le coefficient $C_{T}$ prend en valeur 1 et les apports en azote et phosphore d'un bassin versant s'écrivent :

$$
F=\Sigma F_{1}=\Sigma P_{1}+C_{1} \Sigma D_{1}
$$

où :

$F_{1}$ représente le flux journalier moyen transité pour l'élément i. Les coefficients $C_{\mathrm{I}}$ ont été établis à $15 \%$ et $5,6 \%$ pour l'azote et le phosphore respectivement; la différence entre ces coefficients de transfert est attribuable essentiellement à des considérations de mobilités chimiques et biochimiques de l'azote et du phosphore.

\section{3. - RESULTATS ET DISCUSSION}

\section{1. - Les charges en azote et en phosphore}

La connaissance de la distribution des superficies occupées par la forêt et des surfaces exploitées à des fins agricoles permet de distin- 
guer deux grands groupes de tributaires : les rivières forestières et les rivières agricoles. Les sous-bassins à caractère forestier ont des superficies boisées qui occupent entre 91,7 et $99,1 \%$ de leur territoire ; ce sont les bassins des rivières Péribonca, Mistassini, Chamouchouane, Petite Péribonca, Métabetchouane, Ouiatchouane et Ouiatchouaniche. Les sous-bassins agricoles bien qu'ayant des surfaces de forêts couvrant entre $38,9 \%$ et $70 \%$ de leur territoire ont des superficies réservées à des activités agricoles qui représentent entre 22 et $41 \%$ de leur surface totale; les tributaires considérés ici sont les rivières Ticouapé, Mistouc, Des Chicots, Moreau et Couchepaganiche.

Le calcul des charges transitées à l'intérieur des tributaires montrent que les trois grandes rivières forestières (Péribonca, Mistassini et Métabetchouane) transportent l'essentiel des charges d'azote et de phosphore (Tableaux III et IV). Les tributaires agricoles transitent en général des charges moins élevées compte tenu de leur faible débit. Le calcul des contributions relatives de chaque secteur de production (forêt, agriculture et population) effectué à partir des statistiques concernant l'état ou l'utilisation des territoires drainés identifie bien les sous-bassins où l'origine des apports est essentiellement naturelle (les rivières forestières) et les sous-bassins où les activités humaines sont importantes (rivières agricoles).

TABLEAU III. - Répartition des charges en azote dans les principaux tributaires du lac Saint-Jean.

\begin{tabular}{lrrrrr}
\hline & $\begin{array}{c}\text { APPORTS } \\
\text { TOTAUX } \\
\mathrm{kg} / \text { jour }\end{array}$ & $\begin{array}{c}\text { Forêt } \\
\%\end{array}$ & $\begin{array}{c}\text { ORIGINE DES APPORTS } \\
\text { Agriculture } \\
\%\end{array}$ & $\begin{array}{c}\text { Population } \\
\%\end{array}$ & $\begin{array}{c}\text { DENSITÉ } \\
\text { DE CHARGES } \\
\mathrm{g} / \mathrm{km}^{2} / \mathrm{jour}\end{array}$ \\
\hline $\begin{array}{l}\text { a) Rivières forestières } \\
\text { Féribonca }\end{array}$ & 21281 & & & & \\
Mistassini & 17558 & 99,7 & 0,2 & 0,07 & 790 \\
Chamouchouane & 12389 & 98,0 & 0,9 & 1,0 & 800 \\
Petite Péribonca & 977 & 98,0 & 1,0 & 0,9 & 800 \\
Métabetchouane & 1876 & 92,0 & 7,0 & 0,6 & 830 \\
Ouiatchouane & 786 & 97,0 & 1,6 & 0,8 & 820 \\
Ouiatchouaniche & 297 & 94,0 & 2,9 & 3,5 & 820 \\
& 86,0 & 12,0 & 1,8 & 840 \\
b) Rivières agricoles & & & & & \\
Ticouapé & 948 & 22,0 & 72,0 & 6,0 & 1430 \\
Mistouc & 233 & 36,0 & 57,0 & 6,0 & 1090 \\
Des Chicots & 71 & 37,0 & 61,0 & 1,9 & 1390 \\
Moreau & 116 & 49,3 & 49,8 & 0,9 & 1045 \\
Couchepaganiche & 153 & 37,0 & 50,0 & 13,0 & 1490 \\
\hline
\end{tabular}

Les densités de charges obtenues à partir des apports totaux et des surfaces drainées mettent en évidence l'intensité de l'utilisation du sol; ce sont évidemment les tributaires à caractère agricole qui ont 
TableaU IV. - Répartition des charges en phosphore dans les principaux tributaires du lac Saint-Jean.

\begin{tabular}{|c|c|c|c|c|c|}
\hline & APPORTS & & RIGINE DES APP & ORTS & DENSITÉ \\
\hline & $\begin{array}{l}\text { TOTAUX } \\
\mathrm{kg} / \text { jour }\end{array}$ & $\begin{array}{c}\text { Forêt } \\
\%\end{array}$ & $\begin{array}{c}\text { Agriculture } \\
\%\end{array}$ & $\begin{array}{c}\text { Population } \\
\%\end{array}$ & $\begin{array}{l}\text { DE CHARGES } \\
\mathrm{g} / \mathrm{km}^{2} / \text { jour }\end{array}$ \\
\hline a) Rivières for & tières & & & & \\
\hline Péribonca & 967 & 99 & 0,4 & 0,2 & 36 \\
\hline Mistassini & 818 & 95 & 1,9 & 2,8 & 37 \\
\hline Chamouchouane & 579 & 96 & 1,8 & 2,5 & 37 \\
\hline Petite Péribonca & 475 & 86 & 13,0 & 1,2 & 40 \\
\hline Métabetchouane & 87,1 & 95 & 2,8 & 2,1 & 37 \\
\hline Ouiatchouane & 38,5 & 86 & 4,4 & 9,1 & 40 \\
\hline Ouiatchouaniche & 14,7 & 79 & 19,0 & 1,8 & 42 \\
\hline b) Rivières agri & & & & & \\
\hline Ticouapé & 80,3 & 12 & 80 & 8,0 & 120 \\
\hline Mistouc & 14,9 & 23 & 66 & 11,0 & 70 \\
\hline Des Chicots & 4,3 & 22 & 76 & 1,3 & 100 \\
\hline Moreau & 7,9 & 30 & 62 & 0,9 & 71 \\
\hline Couchepaganiche & 11,2 & 22 & 56,0 & 11,2 & 110 \\
\hline
\end{tabular}

les densités les plus élevées par rapport aux rivières forestières. Les valeurs du rapport des charges calculées en azote et en phosphore $(\mathrm{N} / \mathrm{P})$ montrent que les rivières agricoles sont plus chargées en nutriments que les tributaires forestiers: $\mathrm{N} / \mathrm{P}_{\text {(agricole) }}<\mathrm{N} / \mathrm{P}_{\text {(eorestier) }}$ (Tableau $\mathrm{V}$ ). Les rapports $\mathrm{N} / \mathrm{P}$ obtenus sont semblables à ceux calculés à partir des données fournies par Lee et al. (1978) concernant des densités de charges de bassins agricoles ou forestiers situés aux EtatsUnis.

TABLEAU V. - Rapport N/P des charges (en équivalent atomique).

\begin{tabular}{lrrr} 
& $\mathrm{N}$ & $\mathrm{P}$ & $\mathrm{N} / \mathrm{P}$ \\
\hline a) Rivières forestières & & \\
Péribonca & 56,4 & 1,2 & 47,0 \\
Mistassini & 57,1 & 1,2 & 47,6 \\
Chamouchouane & 57,1 & 1,2 & 47,6 \\
Petite Péribonca & 59,3 & 1,3 & 45,6 \\
Métabetchouane & 57,8 & 1,2 & 48,2 \\
Ouiatchouane & 58,6 & 1,3 & 45,1 \\
Ouiatchouaniche & 60,0 & 1,4 & 42,9 \\
b) Rivières agricoles & & \\
Ticouapé & 102,1 & 3,9 & 26,2 \\
Mistouc & 77,9 & 2,3 & 33,9 \\
Des Chicots & 99,3 & 3,2 & 31,0 \\
Moreau & 74,6 & 2,3 & 32,4 \\
Couchepaganiche & 106,4 & 3,5 & 30,4 \\
\hline
\end{tabular}




\section{2. - La fertilité des eaux et les concentrations en chlorophylle}

Les variations du PF (Tableau VI) traduisent différentes concentrations en substances nutritives disponibles qui sont caractérisées à l'aide des indices d'enrichissement. Ces derniers ont été définis à partir de la notion des indices de productivité déterminés par Miller et al. (1974) pour des lacs et appliqués en rivière par Greene et al. (1975 a). Dans le cas des tributaires étudiés, les indices varient entre 2 et 4 ; cette situation indique un niveau d'enrichissement en substances nutritives disponibles comparable à celui retrouvé dans des eaux mésotrophes (indice 2) ou eutrophes (indice 3 ou 4). Les indices 3 et 4 sont habituellement liés aux rivières agricoles; la rivière Ticouapé, par exemple, dont le sous-bassin occupe la plus importante superficie utilisée à des fins agricoles dans le bassin du lac Saint-Jean, se distingue nettement des autres tributaires par la valeur élevée (4) de son indice. Quant aux rivières forestières, elles sont caractérisées par des valeurs moins élevées de l'indice qui, dans la plupart des cas, prend la valeur 2 .

Tableau VI. - Potentiels de fertilité et indices d'enrichissement des tributaires.

\begin{tabular}{lcccccccc}
\hline & \multicolumn{2}{c}{22 mai } & \multicolumn{2}{c}{17 juin } & \multicolumn{2}{c}{15 juillet } & \multicolumn{2}{c}{23 sept. } \\
& PF 1 & IE 2 & PF & IE & PF & IE & PF & IE \\
\hline \multicolumn{2}{l}{ a) Rivières forestières } & & & & & & & \\
Péribonca & 0,1 & 2 & 0,6 & 2 & 0,6 & 2 & 0,3 & 2 \\
Mistassini & 0,3 & 2 & 1,2 & 3 & 0,3 & 2 & 0,5 & 2 \\
Chamouchouane & 0,2 & 2 & 1,0 & 3 & 0,2 & 2 & 0,4 & 2 \\
Petite Péribonca & 0,8 & 2 & 1,4 & 3 & 0,5 & 2 & 0,6 & 2 \\
Métabetchouane & 0,6 & 2 & 1,2 & 3 & 0,5 & 2 & 1,4 & 3 \\
Ouiatchouane & 0,5 & 2 & 0,9 & 3 & 0,4 & 2 & 0,5 & 2 \\
Ouiatchouaniche & 0,9 & 3 & 1,4 & 3 & 0,6 & 2 & & \\
\multicolumn{1}{l}{ b) Rivières agricoles } & & & & & & & \\
Ticouapé & 15,0 & 4 & 13,0 & 4 & 13,0 & 4 & 9,0 & 4 \\
Mistouc & 2,9 & 3 & 3,7 & 3 & 1,3 & 3 & 1,1 & 3 \\
Des Chicots & 3,2 & 3 & 3,3 & 3 & 0,7 & 2 & 1,0 & 3 \\
Moreau & 2,1 & 3 & 5,0 & 3 & 3,0 & 3 & 1,8 & 3 \\
Couchepaganiche & 1,1 & 3 & 1,9 & 3 & 0,6 & 2 & 1,4 & 3 \\
\hline
\end{tabular}

$1 \mathrm{PF}$ : potentiel de fertilité en $\mathrm{mg}$ d'algues/1.

2 IE : indice d'enrichissement : 1 : faible : $<0,1 \mathrm{mg}$ d'algues $/ 1$

2: moyen : $0,1 \cdot 0,8 \mathrm{mg}$ d'algues $/ 1$

3 : modérément élevé : $>0,8-6 \mathrm{mg}$ d'algues $/ 1$

$4:$ élevé $:>6 \mathrm{mg}$ d'algues $/ \mathrm{l}$.

Il est intéressant de constater que l'ensemble des PF mesurés sur les rivières agricoles présente des valeurs supérieures à $1 \mathrm{mg}$ d'al- 
gues par litre. La plupart des mesures obtenues pour les rivières forestières sont cependant inférieures à cette valeur. Katko (1975) considère qu'une telle valeur $(1 \mathrm{mg} / \mathrm{l})$ est le reflet d'un niveau d'enrichissement acceptable; les valeurs supérieures pourraient traduire une situation problématique.

En ce qui concerne les concentrations en chlorophylle- $a$ totale (Tableau VII), les rivières agricoles ont tendance à supporter des concentrations plus élevées que celles qui ont été mesurées dans les rivières forestières. Cette situation semble refléter les niveaux d'enrichissement des différents groupes de rivières; en effet, l'analyse des indices d'enrichissement a déjà mis en évidence un potentiel de production plus élevé pour les tributaires localisés dans les régions agricoles. Il faut toutefois signaler que la relation niveau d'enrichissement $\leftrightarrow$ concentration en chlorophylle peut être affectée par différents facteurs comme la température, l'effet du broutage, la turbidité et la vitesse du courant. La présence de tels facteurs entraînera nécessairement des différences sur la production phytoplanctonique du milieu, même si les potentiels de production établis devaient s'avérer semblables.

TABLEAU VII. - Concentration en chlorophylle- $a$ totale des tributaires $(\mu \mathrm{g} / \mathrm{l})$.

\begin{tabular}{lccc}
\hline & 17 juin & 15 juillet & 23 septembre \\
\hline \multicolumn{2}{c}{ a) Rivières forestières } & & \\
Péribonca & 0,22 & 2,53 & 0,58 \\
Mistassini & 0,42 & 1,10 & 0,28 \\
Chamouchouane & 0,61 & 1,46 & 0,35 \\
Petite Péribonca & 0,53 & 1,14 & 0,55 \\
Métabetchouane & 0,89 & 3,08 & 1,06 \\
Ouiatchouane & 1,87 & 2,09 & 2,01 \\
Ouiatchouaniche & 1,16 & 1,13 & 1,32 \\
b) Rivières agricoles & & \\
Ticouapé & 3,05 & 23,94 & 1,83 \\
Mistouc & 0,99 & 2,09 & 2,36 \\
Des Chicots & - & 3,53 & 3,13 \\
Moreau & 1,12 & 4,20 & 0,85 \\
Couchepaganiche & 0,70 & 7,97 & 0,27 \\
\hline
\end{tabular}

\section{3. - Les charges calculées et leur biodisponibilité}

Les considérations des valeurs de $\mathrm{PF}$ confrontées aux rapports $\mathrm{N} / \mathrm{P}$ mettent en évidence les deux groupes de stations (fig. 2). Les rivières forestières ont des rapports $\mathrm{N} / \mathrm{P}$ plus élevés que les rivières agricoles ; 


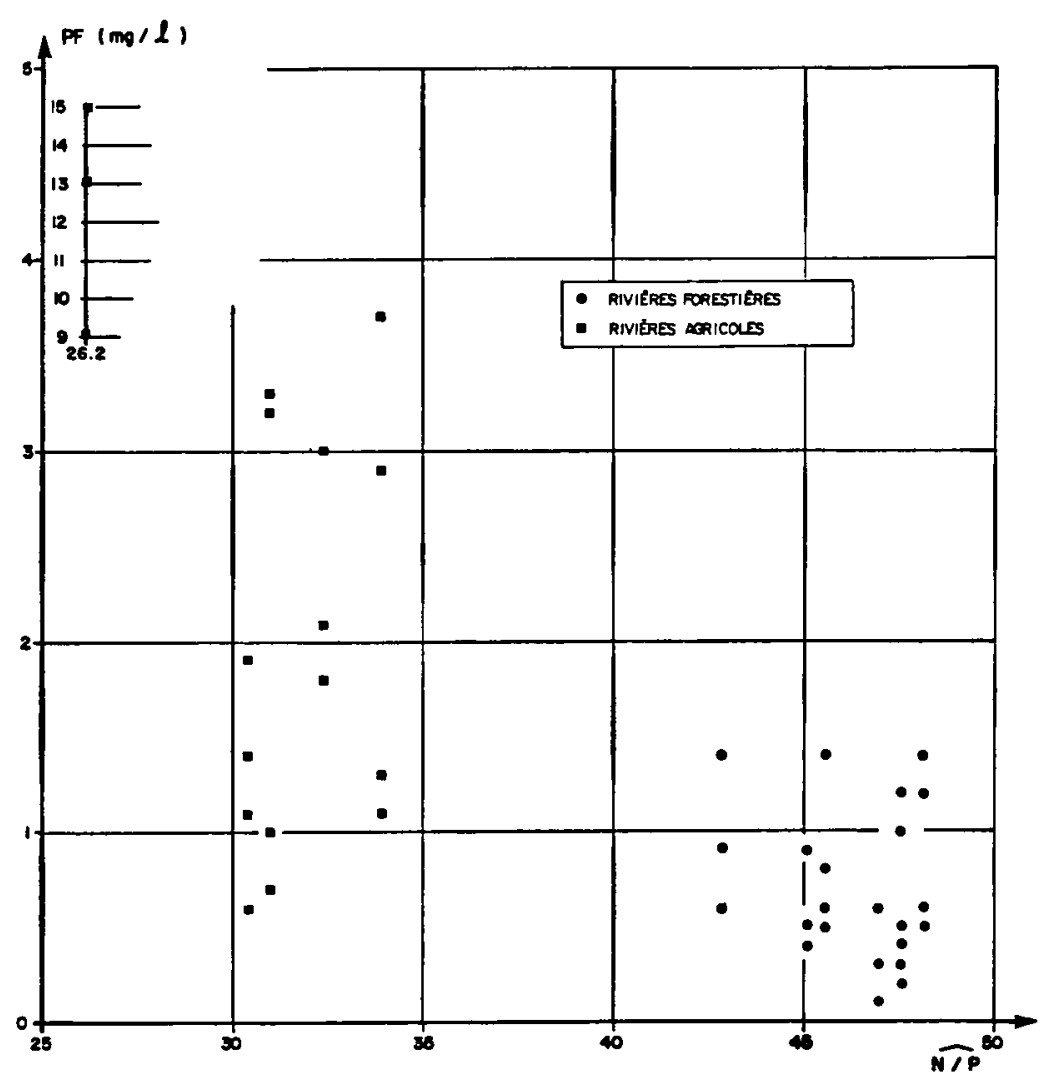

FIG. 2. - Typologie des tributaires.

par contre, les concentrations en substances nutritives disponibles s'avèrent, en général, plus faibles dans les rivières forestières. Il est reconnu (Greene et al. 1975 a ; INRS-Eau 1976; Miller et al. 1974 ; Robarts et Southall 1977) que pour les eaux de surface, le rapport des milieux enrichis (eutrophes) est inférieur à celui des milieux naturels pauvres en éléments nutritifs (oligotrophes).

La rivière Ticouapé se distingue des rivières agricoles tant par ses concentrations en éléments nutritifs disponibles que par les valeurs du rapport $\mathrm{N} / \mathrm{P}$. De plus, on constate que malgré des PF nettement plus élevés que les autres tributaires agricoles, le rapport n'est pas davantage affecté. Cette situation serait due au fait que les activités agricoles responsables de $72 \%$ des apports en azote et de $80 \%$ des apports en phosphore sont considérées comme des sources diffuses lors de l'application du modèle d'apport. Cette conception a pour effet de minimiser l'importance des charges en phosphore par rapport aux charges en azote, ce qui se traduit par une augmentation du rapport; on se souviendra que dans le cas des apports diffus, les coefficients de 
transfert ont été établis à $15 \%$ et $5,6 \%$ pour l'azote et le phosphore respectivement. Or, il s'avère que compte tenu de l'intensité et du type d'élevage pratiqué sur ce bassin versant, les apports devraient ètre considérés comme ponctuels ; cette considération sur la nature ponctuelle des rejets d'élevages industriels aurait pour effet de réduire la valeur du rapport à 11 et de discriminer davantage le sous-bassin de la rivière Ticouapé par rapport aux autres sous-bassins agricoles.

\section{4. - CONCLUSION}

Le calcul des charges en éléments nutritifs ainsi que l'évaluation de leur biodisponibilité ont contribué à établir une typologie des deux groupes de sous-bassins étudiés; en effet, la considération simultanée des rapports N/P et des PF a mis en évidence que ces deux variables permettent de distinguer les tributaires agricoles des rivières forestières.

Il faut noter que selon cette typologie, les rivières forestières ont toujours des PF inférieurs à 1,5 et des rapports $N / P$ variant entre 43 et 48 alors que les tributaires agricoles ont des PF plus élevés et des rapports $N / P$ variant entre 30 et 34 . Cette discrimination, résultant de l'utilisation et de l'état des territoires drainés, est représentative du bassin du lac Saint-Jean.

D'autres recherches seraient nécessaires pour vérifier, sur des bassins d'utilisation plus diversifiée si le rapport $\mathrm{N} / \mathrm{P}$ permet toujours de discriminer les types d'usage tout en demeurant un indice d'enrichissement des écosystèmes aquatiques.

\section{TRAVAUX CITES}

Berland (B. R.), Bonin (D. J.) et Maestrini (S. Y.). 1974. - Mise en évidence de facteurs réglant la productivité du phytoplancton des eaux de surface dans la partie nord occidentale de la mer Méditerranée au moyen de tests biologiques. Ann. Inst. Océanogr., Paris, 50: 5-25.

Berland (B. R.), Bonin (D. J.) et Maestrini (S. Y). 1976. - De l'emploi concomitant d'enceintes dialysantes et de tests biologiques pour la détermination des facteurs nutritionnels limitant la production primaire des eaux marines. Ann. Inst. Océanogr., Paris, 52: 45-55.

Chiaudani (G.) et Vighi (M.). 1974. - The N:P ratio and tests with Selenastrum to predict eutrophication in lakes. Water Res., $8: 1063-1069$.

Cluis (D.), Couillard (D.) et Potvin (L.). 1979. - A square grid transport model relating land-use exports to nutrient loads in rivers. Water Res., 15: 630-636.

Dillon (P. J.) et Kirchner (N. B.). 1975. - The effects of geology and land use on the export of phosphorous from watersheds. Water Res., $9: 135-148$. 
Dillon (P. J.) et Rigler (F. H.). 1975. - A simple method for predicting the capacity of a lake for development based on lake trophic status. J. Fish. Res. Bd Can., 32 : 1519-1531.

DoEmel (W. N.) et BrooKs (A. E.). 1975. - Detergent phosphorous and algal growth. Water Res., $9:$ 713-719.

EPA. (1971). - Provisional algal assay procedures. Final report. Sanitary Engineering Research Laboratory, College of Engineering and School of Public Health, University of California, Berkeley SERL Report No 71-6, 211 p.

Francisco (D. F.) et WeIss (C. M.). 1973. - Algal response to detergent phosphate levels. J. Water Pollut. Cont. Fed., $45: 480-489$.

Greene (J. C.), Milder (W. E.) et Shiroyama (T.). 1975 a. - Utilization of algal assays to assess the effects of municipal, industrial and agricultural wastewater effluents upon phytoplankton production in the Snake River System. Water Air Soil Pollut., 4 : 415-434.

Greene (J. C.), Soltero (R. A.), Miller (W. E.), Gasperino (A. F.) et Shiroyama (T.). $1975 \mathrm{~b}$. - The relationship of laboratory algal assays to measurements of indigenous phytoplankton in Long Lake, Washington. Biostimulation and Nutrient Assessment, Proceeding of a Workshop held at Utah State University, Sept. 1-12, 1975, p. 93-126.

Greene (J. C.), Miller (W. E.), Shiroyama (T.), Soltero (R. A.) et Putman (K.). 1976 a. - Use of algal assays to assess the effects of municipal and smelter wastes upon phytoplankton production. Proceedings of the Symposium on terrestrial and Aquatic Ecological Studies of the Northwest, March 26-27, 1976 : 327-335.

Greene (J. C.), Miller (W. E.), Shiroyama (T.), Soltero (R. A.) et Putman (K.). $1976 \mathrm{~b}$. - Use of laboratory cultures of Selenastrum, Anabaena and the indigenous isolate Sphaerocystis to predict effects of nutrient and zinc interactions upon phytoplankton growth in Long Lake, Washington. International Symposium on Experimental Use of Algal Cultures in Limnology, Oct. 26-28, 1976.

GeRhold (R. M.) et Otto (R. G.). 1976. - Algal bioassays to evaluate a proposed cooling lake. J. Water Pollut. Cont. Fed., $48:$ 2351-2366.

INRS-EAU (Institut national de la recherche scientifique - Eau). 1976. - Etude intégrée de la qualité des eaux des bassins versants des rivières Saint-François et Yamaska. Volume 2: Secteur des substances nutritives. (INRS-Eau, Rapport scientifique $\mathrm{n}^{\circ} 52$ ), INRS, Québec, $85 \mathrm{p}$.

INRS-EAU (Institut National de la recherche scientifique - Eau). 1980. - Productivité biologique des eaux du lac Saint-Jean. (INRS-Eau, Rapport scientifique $\mathrm{n}^{\circ} 76$ ), INRS, Québec, $603 \mathrm{p}$.

KatKo (A.). 1975. - Algal assay fort the national eutrophication survey. Biostimulation and Nutrient Assessment, Proceedings of a Worksphop, Oct. 16-17, 1973 , p. $44-52$.

KIRCHNER (W. B.). 1975. - An examination of the relationship between drainage basin morphology and the export of phosphorus. Limnol. Oceanogr., 20 : 267-270.

LEE (F. G.). 1973. - Role of phosphorus in eutrophication and diffuse source control. Water Res., $7:$ 111-128.

LeE (F. G.), RAST (W.) et JoNES (R. A.). 1978. - Eutrophication of water bodies: insights for an age-old problem. Env. Sci. Tech., 12 : 900-908.

Likens (G. E.) et Loucks (O. L.). 1978. - Analysis of five North American lake ecosystems. III : Sources, loading and fate of nitrogen and phosphorous. Verh. Internat. Verein. Limnol., 20 : 568-573.

MAHONEY (J. L.). 1973. - Shagawa Lake project report. U.S. Environmental Protection Agency, Eutrophication and Lake Restoration. Branch, Corvallis, Oregon (unpublished report). 
Middlebrooks (E. J.), Porcella (D. B.), Pearson (E. A.), Mc Gauhey (P. H.) et RoHLICH (G. A.). 1971. - Biostimulation and algal growth kinetics of wastewater. J. Water Pollut. Cont. Fed., 43 : 454-473.

Miller (W. E.), Maloney (T. E.) et Greene (J. C.). 1974. - Algal productivity in 49 lake waters as determined by algal assays. Water Res., $8: 667-679$.

Mitchell (D.) et Buzzell Jr. (J. C.). 1971. - Estimating eutrophic potential of pollutants. J. Sanit. Eng. Div. Proc. Am. Soc. Civ. Eng., (SA4) : 453-465.

MRN (Ministère des Richesses naturelles). 1977. - Etude intégrée de la qualité des eaux des bassins versants des rivières Saint-François et Yamaska. Volume 3: Secteur du modèle d'apports. MRN, Service qualité des eaux, Québec. $105 \mathrm{p}$.

Parsons (T. R.) et STRickland (J. D.). 1972. - Practical handbook of seawater analysis. Fisheries Research Board of Canada, Ottawa, $310 \mathrm{p}$.

I'atalas (K.) et Salki (A.). 1973. - Crustacean plankton and the eutrophication of lakes in the Okanaga Valley, B.C. J. Fish. Res. Bd Can., $30: 519-542$.

PaYNe (A. G.). 1973. - Environmental testing of citrate : bioassays for algal stimulation. Proceedings 16th Conference Great Lakes Research. International Association Great Lakes Research, p. 100-115.

Payne (A. G.). 1975. - Responses of the three test algae of the algal assay procedure : bottle test. Water Res., 9: 937-455.

Robarts (R. D.) et Southall (G. C.). 1977. - Nutrient limitation of phytoplankton growth in seven tropical man-made lakes, with special reference to lake McIlwaine, Rhodesia. Arch. Hydrobiol., 79: 1-35.

Scorr's. 1975. - Répertoire industriel du Québec. 7c édition. Penstock Publications Ltd.

Steyn (D. J.), Toerien (D. F.) et Visser (J. H.). 1974. - Continuous culture algal bioassays. South African J. Sci., $70: 277-278$.

Sturm (R. N.) et Payne (A. G.). 1973. - Environmental testing of trisodium nitrilotriacetate : bioassays for aquatic safety and algal stimulation. Bioassay Techniques and Environmental Chemistry. Glass, G.E. (ed). Ann Arbor Science Publishers Inc., p. 403-424.

Thomas (W. H.), Seibert (D L. R.) et Dodson (A. N.). 1974. - Phytoplankton enrichment experiments and bioassays in natural coastal sea water and in sewage outfall receiving waters off Southern California. Estuar. Coast. Mar. Sci., 2: 191-206.

TOERIEN (D. F.) et STEYN (D. J.). 1973. - Application of algal bioassays in eutrophication analyses. South African J. Sci., $69: 79-82$.

VOLLENWEIDER (R. A.). 1968. - Les bases scientifiques de l'eutrophisation des lacs et des eaux courantes sous l'aspect particulier du phosphore et de l'azote comme facteurs d'eutrophisation. OCDE, Paris, rapport DAS/CSI/C8.27 : 95-148.

Werss (C. M.). 1975. - Field evaluation of the algal assay procedure on surface waters of North Carolina. Biostimulation and Nutrient Assessment, Proceedings of a Workshop held at Utah State University, Sept., 10-12, 1975 : 29-76. 